Matheus Oliveira-Abreu ${ }^{1}$, Mônica Lajana de Almeida²

1. Especialista em Fisioterapia Cardiorrespiratória pelo Instituto do Coração (InCor) do Hospital das Clínicas da Faculdade de Medicina da Universidade de São Paulo - USP- São Paulo (SP), Brasil.

2. Professora da Faculdade Social e da União Metropolitana de Educação e Cultura - UNIME - Salvador (BA), Brasil.

Recebido da União Metropolitana de Educação e Cultura -UNIME Salvador (BA), Brasil.

Submetido em 8 de Junho de 2008 Aceito em 9 de Março de 2009

Autor para correspondência: Matheus Oliveira Abreu Rua Florentino Silva, 243 - Itaigara CEP: 41815-400 - Salvador (BA), Brasil. Fone: (11) 8539-4667

E-mail: matheusabr@aol.com

\section{Manuseio da ventilaçáo mecânica no trauma cranioencefálico: hiperventilação e pressão positiva expiratória final}

\author{
Management of mechanical ventilation in brain injury: \\ hyperventilation and positive end-expiratory pressure
}

\section{RESUMO}

O objetivo desse artigo foi realizar uma revisão de literatura sobre a utilização de manobras de hiperventilação pulmonar e os níveis de pressão positiva expiratória final empregados em pacientes com trauma cranioencefálico. Foram utilizados como referências publicações em inglês, espanhol e português, contidas nas bases de dados: MedLine, SciELO e LILACS, de 2000 até 2007, tendo como critérios de inclusão: manobra de hiperventilação pulmonar e o nível de pressão positiva expiratória final aplicado ao paciente adulto com trauma cranioencefálico, agudo ou crônico. Foram selecionados 31 estudos, sendo 13 sobre a utilização da hiperventilação pulmonar, como profilática, prolongada ou otimizada e 9 abordavam o emprego da pressáo positiva expiratória final, em níveis que variaram de 0 a $15 \mathrm{cmH}_{2} \mathrm{O}$. A hiperventilação profilática nas primeiras 24 horas pode levar a um aumento da isquemia cerebral; a hiperventilação prolongada deve ser evitada na ausência de elevada pressão intracraniana; já a hiperventilação otimizada parece ser a técnica mais promissora no controle da pressão intracraniana e da pressão de perfusão cerebral. A elevação da pressão positiva expiratória final até 15 $\mathrm{cmH}_{2} \mathrm{O}$ pode ser aplicada, de forma consciente, com o objetivo na elevação da saturação arterial de oxigênio em presença de injúria pulmonar.

Descritores: Traumatismos craniocerebrais; Hipertensão intracraniana; Pressão intracraniana; Respiração com pressão positiva; Hiperventilação

\section{INTRODUÇÁO}

O trauma cranioencefálico (TCE) representa a principal causa mundial de morbimortalidade em indivíduos de idade inferior a 45 anos, com maior predominância no sexo masculino. Ocorre em cerca de $40 \%$ das vítimas de trauma, sendo que $20 \%$ delas falecem no local ou no primeiro dia de internação e $80 \%$ entre os primeiros sete dias posteriores ao evento. ${ }^{(1-3)}$

O TCE é uma lesão de natureza não degenerativa ou congênita, causada por uma agressão ou iniciada por um processo de aceleração ou desaceleração de alta energia do cérebro dentro do crânio, que gera um dano anatômico ou comprometimento funcional do couro cabeludo, crânio, meninges ou encéfalo. ${ }^{(4,5)}$ Pode ser provocado por acidentes de trânsito, quedas, agressōes, perfuração por arma branca ou de fogo, grandes catástrofes e atividades esportivas. ${ }^{(6)}$ Quando apropriado, devese pesquisar drogas e álcool no organismo. ${ }^{(7)}$

Dois diferentes mecanismos determinam a gravidade do traumatismo: (1) primeiro insulto, que ocorre no momento do impacto. (2) segundo insulto, que representa um processo patológico consecutivo às alteraçóes clínicas iniciais ao trauma..$^{(1)}$ 
O rebaixamento do nível de consciência é o principal fator de risco para a broncoaspiração e posterior admissáo na unidade de tratamento intensivo (UTI), que tem como objetivos detectar e tratar as complicaçôes da lesão primária e fornecer uma melhor condição para o retorno da funçáo cerebral. ${ }^{(8)}$ Portanto, pacientes com problemas relacionados ao sistema nervoso central (SNC) muitas vezes necessitam de assistência ventilatória por insuficiência respiratória aguda (IRpA), nem sempre pela condição neurológica em si, como a diminuiçáo do drive respiratório, mas por afecçốes pulmonares. ${ }^{(9)}$

A ventilação mecânica constitui um dispositivo terapêutico imprescindível em pacientes com TCE grave, uma vez que visa a proteção da via aérea, pela intubação endotraqueal, e permite a sedação, inclusive curarização, evitando assim os danos causados pela hipoxemia e hipercapnia. ${ }^{(10)}$

Baseada nas terapêuticas ventilatórias adotadas nos últimos anos em pacientes com TCE, principalmente os que apresentam hipertensão intracraniana (HIC), esta revisão teve o intuito de comparar as diferentes técnicas ventilatórias empregadas no manuseio dos pacientes com TCE e as repercussóes nos parâmetros de monitorização neurológica.

\section{MÉTODOS}

Foi realizada uma revisão da literatura sobre o TCE, tendo como referências publicações em inglês, espanhol e português, cujos descritores foram trauma cranioencefálico, hipertensão intracraniana, pressão intracraniana, pressão positiva expiratória final (PEEP) e hiperventilação, contidas nas bases de dados: MedLine, SciELO e LILACS, publicados entre 2000 e 2007. Foram selecionados estudos que abordavam diferentes níveis de PEEP e a utilizaçáo de manobras de hiperventilação pulmonar no paciente adulto com TCE, agudo ou crônico, assim como suas repercussôes nos parâmetros neurológicos

Para avaliação dos estudos foram estabelecidos critérios, de forma que se pudesse assegurar a qualidade dos trabalhos, como: (a) identificação do estudo quanto ao tipo de tratamento e como se procede a técnica; (b) característica metodológica dos ensaios clínicos ou dos artigos de revisão. $\mathrm{Na}$ avaliação dos atributos qualitativos, foram levadas em conta a redução ou elevaçáo da pressão intracraniana (PIC), assim como normalização da pressão de perfusão cerebral (PPC) e da pressão arterial média (PAM).

\section{FISIOPATOLOGIA DO TRAUMATISMO CRÂ- NIO ENCEFÁLICO}

Os mecanismos do TCE provocam disrupturas na barreira hemato-encefálica permitindo que os componentes plas- máticos atravessem facilmente essa barreira para dentro do tecido neural (edema vasogênico). A hipóxia (injúria secundária) afeta a ATPase sódio/potássio da membrana celular, promovendo acúmulo intracelular de sódio, e o subseqüente fluxo de água para dentro da célula por gradiente osmótico. Logo, o edema citotóxico também ocorre, porém em fase subaguda e/ou crônica. Portanto, o edema vasogênico, acrescido de possíveis áreas localizadas de hemorragias com efeito de massa, são os principais responsáveis pelo surgimento da hipertensão intracraniana. Tais mecanismos atingem o pico em torno de três a cinco dias. ${ }^{(11,12)}$

Mudanças no fluxo cerebral, inflamação e edema são componentes da patogênese das alteraçóes nos tecidos cerebrais. $\mathrm{O}$ cérebro fica contido por uma estrutura rígida, não complacente, onde um grau relativamente pequeno de tecido edemaciado pode aumentar a PIC. Este também possui um sistema especial de auto-regulação do fluxo sanguíneo cerebral (FSC), que é mantido, em condiçóes normais, mesmo com PAM variando entre 50 e $140 \mathrm{mmHg}$. A auto-regulação do FSC é conseguida pela rápida constricção e relaxamento das arteríolas cerebrais e vênulas, em resposta aos fatores químicos e endoteliais e a liberação de neurotransmissores de neurônios adjacentes. ${ }^{(8)}$

O FSC depende da diferença da pressão arterial e a pressão venosa cerebral, sendo inversamente proporcional a resistência vascular cerebral. A PPC é calculada pela diferença entre a PAM e a PIC. A PPC de $60 \mathrm{mmHg}$ é comumente aceita como o valor mínimo necessário para uma adequada perfusáo cerebral. ${ }^{(11-13)}$ A PIC é determinada pela pressão do parênquima cerebral, volume sanguíneo cerebral e volume de líquor $^{(3)}$. O aumento da PIC é comum após o TCE, quando a complacência intracraniana não consegue acomodar o aumento do volume. O valor normal da PIC em adultos é de $10 \mathrm{mmHg}$, valores acima de $20 \mathrm{mmHg}$ são indicativos de intervenção terapêutica. Valores entre 10 e 20 são considerados levemente aumentados e entre 20 e $40 \mathrm{mmHg}$, moderadamente elevados. Acima de $40 \mathrm{mmHg}$ encontram-se os casos graves de hipertensão intracraniana, quando herniações do tecido nervoso podem ocorrer. ${ }^{(8,14)}$

A hiperventilação pode reduzir a PIC pela hipocapnia que induz a vasoconstricção cerebral com conseqüente redução do FSC. A induçáo rotineira de vasoconstricçáo pela hipocapnia pode provocar uma reduçáo adicional do FSC, o que agravaria o déficit perfusional, levando, posteriormente, a uma isquemia cerebral. ${ }^{(9,15-17)}$

A PEEP aumenta a capacidade residual funcional (CRF), previne o uso de alta fração inspirada de oxigênio $\left(\mathrm{FiO}_{2}\right)$ e pode reduzir a incidência de lesáo induzida pela ventilaçáo mecânica. Porém, pode causar efeitos deletérios no compartimento cerebral pelo aumento da pressáo intratorácica, que 
irá aumentar a pressão venosa central (PVC), influenciando no retorno do fluxo sanguíneo ao coração. Por fim, há uma reduçáo do débito cardíaco (DC), com conseqüente decréscimo da PAM e PPC. ${ }^{(18)}$

\section{REVISÃO DE LITERATURA}

Neste estudo foram selecionadas 31 publicaçóes entre os anos de 2000 e 2007, sendo 11 artigos sobre a utilização da hiperventilação pulmonar ${ }^{(10,13,15-17,19-24)} 7$ sobre a utilização da $\operatorname{PEEP}^{(4,18,26-30)}$, dois abrangendo ambos os temas ${ }^{(14,25)}$ e 11 artigos gerais sobre $\mathrm{TCE}^{(1-3,5-9,911,12,31)}$. Apenas um artigo sobre a hiperventilação pulmonar era clínico ${ }^{(20)}$ enquanto foram encontrados cinco sobre o nível de PEEP. ${ }^{(18,26-29)}$

\section{Hiperventilaçáo pulmonar}

Quanto a hiperventilação pulmonar, todos os nove artigos mencionando a hiperventilação profilática concordam que a mesma não é recomendada nas primeiras 24 horas, pois o FSC está reduzido neste período após o trauma ${ }^{(10,13,16,19,21-25)}$. Seis artigos concluem que a hiperventilaçáo prolongada deve ser evitada na ausência de PIC elevada, uma vez que a vasoconstricção sustentada reduz o FSC a níveis deletérios, podendo gerar isquemia cerebral ${ }^{(10,14,19,21,23,24)}$. Há consenso sobre a hiperventilação otimizada, por curto período em PIC elevada, devendo-se buscar o fator causal da elevação da PIC e tentar tratá-lo; também há consenso sobre o efeito vasoconstrictor cerebral da hipocapnia gerada pela hiperventilação, que reduz o FSC e a PIC (Quadro 1).

Quadro 1 - Resultados da utilização da hiperventilação

\begin{tabular}{|c|c|c|c|c|}
\hline Referência & Estudo & Método & Resultados & Conclusáo \\
\hline $\begin{array}{l}\text { Belda FJ. } \\
2004^{(10)}\end{array}$ & $\mathrm{R}$ & $\begin{array}{l}\text { Hiperventilação profiláti- } \\
\text { ca e hiperventilação pro- } \\
\text { longada. }\end{array}$ & $\begin{array}{l}\text { Ambos podem compro- } \\
\text { meter a PPC. }\end{array}$ & $\begin{array}{l}\text { Deve-se evitar a hiperventilação profilática } \\
\left(\mathrm{PaCO}_{2} \leq 35 \mathrm{mmHg}\right) \text { nas primeiras } 24 \text { horas e a hi- } \\
\text { perventilação prolongada }\left(\mathrm{PaCO}_{2} \leq 25 \mathrm{mmHg}\right) \text { na } \\
\text { ausência de PIC elevada. }\end{array}$ \\
\hline $\begin{array}{l}\text { Stocchetti N } \\
\text { et al. } 2005^{(13)}\end{array}$ & $\mathrm{R}$ & $\begin{array}{l}\text { Indução de hipocapnia } \\
\text { pela hiperventilação. }\end{array}$ & $\begin{array}{l}\text { Redução da PIC pela va- } \\
\text { soconstricção cerebral e } \\
\text { decréscimo do FSC. Baixo } \\
\text { FSC pode gerar isquemia. }\end{array}$ & $\begin{array}{l}\text { Hipocapnia cautelosa a curto prazo controla a redu- } \\
\text { ção na PIC, significando ser uma terapêutica útil. A } \\
\text { hiperventilação profilática parece ser inapropriada. }\end{array}$ \\
\hline $\begin{array}{l}\text { Deem S. } \\
2006^{(14)}\end{array}$ & $\mathrm{R}$ & $\begin{array}{l}\text { Correlação entre o manu- } \\
\text { seio respiratório no TCE e } \\
\text { sua fisiologia. }\end{array}$ & $\begin{array}{l}\text { O efeito da hiperventilação } \\
\text { na PIC é perdido rapida- } \\
\text { mente, entretanto o efeito no } \\
\text { FSC pode ser sustentado. }\end{array}$ & $\begin{array}{l}\text { É prudente evitar longos períodos de hiperventila- } \\
\text { ção, pois pode exacerbar a isquemia cerebral, princi- } \\
\text { palmente nas primeiras } 24 \text { horas. }\end{array}$ \\
\hline $\begin{array}{l}\text { Vincent } \\
\text { JL, Berré J. } \\
2005^{(15)}\end{array}$ & $\mathrm{R}$ & $\begin{array}{l}\text { Manuseio da HIC, do TCE } \\
\text { e do edema cerebral. Além } \\
\text { do controle da PIC, dividi- } \\
\text { do em } 3 \text { intervençóes. }\end{array}$ & $\begin{array}{l}\text { Períodos de hiperventi- } \\
\text { laçáo podem induzir a } \\
\text { algum grau de isquemia } \\
\text { cerebral. }\end{array}$ & $\begin{array}{l}\text { A hiperventilação fica reservada para casos em que } \\
\text { haja herniação cerebral, pois a redução do FSC é } \\
\text { considerada prioritária, no intuito de evitar o exces- } \\
\text { sivo incremento da PIC. }\end{array}$ \\
\hline $\begin{array}{l}\text { Brain Trau- } \\
\text { ma Founda- } \\
\text { tion. } 2007^{(16)}\end{array}$ & $\mathrm{R}$ & $\begin{array}{l}\text { Hiperventilação para re- } \\
\text { duzir a PIC. }\end{array}$ & $\begin{array}{l}\text { Redução da PIC pela va- } \\
\text { soconstricção cerebral e } \\
\text { diminuição do FSC. Risco } \\
\text { de isquemia na hiperventi- } \\
\text { lação agressiva. }\end{array}$ & $\begin{array}{l}\text { A hiperventilação profilática }\left(\mathrm{PaCO}_{2} \leq 25 \mathrm{mmHg}\right) \\
\text { não é recomendada e deve ser evitada nas primei- } \\
\text { ras } 24 \text { hs, devido a prévia redução do FSC. A hiper- } \\
\text { ventilação está recomendada com monitorização da } \\
\text { PIC. }\end{array}$ \\
\hline $\begin{array}{l}\text { Ghajar J. } \\
2000^{(17)}\end{array}$ & $\mathrm{R}$ & $\begin{array}{l}\text { Técnicas de hiperventila- } \\
\text { ção no controle da PIC. }\end{array}$ & $\begin{array}{l}\text { Profilática ou prolongada, } \\
\text { a hiperventilação gera um } \\
\text { pior prognóstico. }\end{array}$ & $\begin{array}{l}\text { A técnica agressiva produz sítios de isquemia cere- } \\
\text { bral. Se após drenagem do líquor a } \mathrm{PIC} \text { permanecer } \\
\text { entre } 20 \text { a } 25 \mathrm{mmHg} \text {, deve-se manter } \mathrm{PaCO}_{2} \text { entre } \\
30 \text { a } 35 \mathrm{mmHg} \text {. Na } \mathrm{HIC} \text { refratária a fármacos e a } \\
\text { procedimentos cirúrgicos, deve-se manter a } \mathrm{PaCO}_{2}< \\
30 \mathrm{mmHg} \text {, com monitorizaçáo da } \mathrm{SjO}_{2} \text { e do } \mathrm{FSC} \text {. }\end{array}$ \\
\hline $\begin{array}{l}\text { Andrade FC, } \\
\text { Andrade Jr } \\
\text { FC. } 2000^{(19)}\end{array}$ & $\mathrm{R}$ & $\begin{array}{l}\text { Manobra de hiperventila- } \\
\text { çáo na prevenção e trata- } \\
\text { mento da HIC. }\end{array}$ & $\begin{array}{l}\text { Provoca isquemia cerebral } \\
\text { em pacientes com TCE } \\
\text { grave. }\end{array}$ & $\begin{array}{l}\text { A manobra de hiperventilação profilática e conti- } \\
\text { nuada devem ser evitadas nos primeiros } 5 \text { dias após } \\
\text { um TCE grave e especialmente proscrita durante as } \\
\text { primeiras } 24 \text { horas. }\end{array}$ \\
\hline
\end{tabular}


Quadro 1 - Continuação

\begin{tabular}{|c|c|c|c|c|}
\hline Referência & Estudo & Método & Resultados & Conclusáo \\
\hline $\begin{array}{l}\text { Falcão ALE, } \\
\text { et al. } 2000^{(20)}\end{array}$ & $\mathrm{E}$ & $\begin{array}{l}\text { Hiperventilação otimizada } \\
\text { em PIC > } 20 \mathrm{mmHg} \text {, con- } \\
\text { trolando a } \mathrm{ECO}_{2} \text { e a PPC. }\end{array}$ & $\begin{array}{l}\mathrm{PaCO}_{2} \text { ideal é aquela que } \\
\text { mantém } \mathrm{PIC}<20 \mathrm{mmHg}, \\
\mathrm{ECO}_{2} \text { entre } 24 \% \text { e } 42 \% \text { e } \\
\mathrm{PPC}>60 \mathrm{mmHg} .\end{array}$ & $\begin{array}{l}\mathrm{O} \text { valor da } \mathrm{PaCO}_{2} \text { é dinâmico e dependente da fase } \\
\text { do TCE, devendo-se dar prioridade à manutenção } \\
\text { de uma adequada } \mathrm{ECO}_{2} \text { para evitar isquemia cere- } \\
\text { bral. }\end{array}$ \\
\hline $\begin{array}{l}\text { Marik PE, } \\
\text { Varon J, } \\
\text { Trask T. } \\
2004^{(21)}\end{array}$ & $\mathrm{R}$ & Manuseio atual do TCE. & $\begin{array}{l}\text { A hiperventilação profilá- } \\
\text { tica foi associada a piores } \\
\text { prognósticos em relação a } \\
\text { normocapnia. }\end{array}$ & $\begin{array}{l}\text { Hiperventilação profilática e prolongada não são } \\
\text { recomendadas. } \mathrm{PaCO}_{2} \text { inicial entre } 35 \text { e } 40 \mathrm{mmHg} \text {. } \\
\text { Curtas hiperventilaçóes, como medida inicial, para } \\
\text { redução da PIC em pacientes com deterioração ce- } \\
\text { rebral. }\end{array}$ \\
\hline $\begin{array}{l}\text { Valadka AB, } \\
\text { Robertson } \\
\text { CS. } 2007^{(22)}\end{array}$ & $\mathrm{R}$ & $\begin{array}{l}\text { Abordagem do uso das } \\
\text { técnicas "positivas" atuais } \\
\text { no TCE. }\end{array}$ & $\begin{array}{l}\text { Pior prognóstico no TCE } \\
\text { grave tratado rotineira- } \\
\text { mente com hiperventila- } \\
\text { ção. }\end{array}$ & $\begin{array}{l}\text { O uso profilático da hiperventilação não é recomen- } \\
\text { dado, tornando-se a última escolha na HIC ou em } \\
\text { nível normal ou elevado do FSC no início da HIC, } \\
\text { assim como na lesão de massa craniana. }\end{array}$ \\
\hline $\begin{array}{l}\text { Vender J. } \\
2000^{(23)}\end{array}$ & $\mathrm{R}$ & $\begin{array}{l}\text { Hiperventilação agressiva, } \\
\text { profilática ou prolongada } \\
\text { no controle da PIC. }\end{array}$ & $\begin{array}{l}\text { A vasoconstricção cere- } \\
\text { bral, redução do FSC e do } \\
\text { volume sanguíneo cerebral } \\
\text { podem rapidamente dimi- } \\
\text { nuir a PIC. }\end{array}$ & $\begin{array}{l}\text { A hiperventilação prolongada }\left(\mathrm{PaCO}_{2}<25 \mathrm{mmHg}\right) \\
\text { deve ser evitada na ausência de elevada } \mathrm{PIC} \text {, assim } \\
\text { como a hiperventilação profilática nas primeiras } 24 \\
\text { h. Porém, a hiperventilação se mantém como terapia } \\
\text { de escolha no tratamento da PIC elevada. }\end{array}$ \\
\hline $\begin{array}{l}\text { Brain Trau- } \\
\text { ma Founda- } \\
\text { tion. } 2000^{(24)}\end{array}$ & $\mathrm{R}$ & $\begin{array}{l}\text { Manuseio e prognóstico } \\
\text { no TCE grave. }\end{array}$ & $\begin{array}{l}\text { A hiperventilação pode } \\
\text { comprometer a perfusão } \\
\text { cerebral em áreas de redu- } \\
\text { ção do FSC. }\end{array}$ & $\begin{array}{l}\text { Na ausência de elevada PIC, a iperventilação prolon- } \\
\text { gada }\left(\mathrm{PaCO} \mathrm{Pa}_{2} \leq 25 \mathrm{mmHg}\right) \text { e a profilática }\left(\mathrm{PaCO}_{2} \leq\right. \\
35 \mathrm{mmHg}) \text { nas primeiras } 24 \text { horas do TCE grave } \\
\text { devem ser evitadas. A hiperventilaçáo pode ser ne- } \\
\text { cessária na deterioração cerebral aguda ou em lon- } \\
\text { gos períodos de HIC refratária a sedação, paralisia, } \\
\text { drenagem do fluido cérebro-espinhal e diuréticos } \\
\text { osmóticos. }\end{array}$ \\
\hline $\begin{array}{l}\text { Helmy A, } \\
\text { Vyzcaychipi } \\
\text { M, Gupta } \\
\text { AK. 2007 } 200\end{array}$ & $\mathrm{R}$ & $\begin{array}{l}\text { Discussão do manuseio } \\
\text { intensivo do TCE grave. }\end{array}$ & $\begin{array}{l}\text { Redução da } \mathrm{PaCO}_{2} \text { causa } \\
\text { vasoconstricçáo cerebral e } \\
\text { diminuição do FSC, que } \\
\text { reduz a PIC. }\end{array}$ & $\begin{array}{l}\text { Não é recomendada a hiperventilação profilática du- } \\
\text { rante as primeiras } 24 \text { horas, se necessária, deve ser } \\
\text { realizada na unidade de tratamento intensivo. }\end{array}$ \\
\hline
\end{tabular}

R- revisão; E - ensaio clínico; $\mathrm{PPC}$ - pressão de perfusão cerebral; $\mathrm{PaCO}_{2}$ - pressão parcial de gás carbônico; PIC - pressão intracranina; $\mathrm{HIC}$ - hipertensão intracraniana; FSC - fluxo sanguineo cerebral; TCE - traumatismo cranioencefálico; SjO2 - saturação venosa jugular de oxigênio; ECO2 - extração cerebral de oxigênio.

Nas primeiras horas após o TCE, os valores absolutos do FSC equiparam-se aos de um evento isquêmico, sendo assim a manobra de hiperventilação nem sempre diminui a PIC para melhorar a PPC, $\mathrm{O}$ valor ideal da $\mathrm{PaCO}_{2}$ é aquele que mantém a PIC $<20 \mathrm{mmHg}$ e a extraçáo cerebral de oxigênio $\left(\mathrm{ECO}_{2}\right)$ entre $24 \%$ e $42 \%$ para evitar isquemia cerebral. ${ }^{(20)}$ A $\mathrm{PaCO}_{2}$ deverá ser mantida entre $35 \mathrm{mmHg}$ e $40 \mathrm{mmHg}$, enquanto que a hiperventilaçáo fica reservada para casos em que haja herniação cerebral. ${ }^{(15,21)}$

Não são recomendadas as manobras de hiperventilação profilática e prolongada durante as primeiras 24 horas, ${ }^{(10,13,14,16,17,19,22-24)}$ ou fora da unidade de tratamento intensivo. $^{(25)}$ A hiperventilação está recomendada em casos que haja monitorização da PIC, ${ }^{(1)}$ sendo indicada por curto período nos episódios de elevação da mesma. Na hiperventilação prolongada, deverão ser monitorizadas a saturaçáo venosa jugular de oxigênio $\left(\mathrm{SjO}_{2}\right)$ e a pressão parcial de oxigênio no tecido cerebral $\left(\mathrm{PbrO}_{2}\right) \cdot{ }^{(14)}$

Dados clínicos demonstraram pior prognóstico no TCE grave tratados rotineiramente com hiperventilaçáo. Existindo duas situaçóes na qual a hiperventilação se torna apropriada: (1) tratamento da HIC de difícil controle; (2) FSC em nível normal ou elevado ao início da HIC. Também, na ocorrência de deterioraçáo cerebral com suspeita de lesão de massa craniana. ${ }^{(22)}$

A hiperventilação pode ser necessária em períodos onde haja deterioração cerebral aguda ou em longos períodos de HIC refratária a sedação, paralisia, drenagem do fluido cérebro-espinhal e diuréticos osmóticos. ${ }^{(24)}$

De forma agressiva, a hiperventilação produz sítios de 
isquemia cerebral. Se após a drenagem do líquor a PIC permanecer entre 20 a $25 \mathrm{mmHg}$, deve-se aplicar a hiperventilação na tentativa de manter $\mathrm{PaCO}_{2}$ entre 30 a $35 \mathrm{mmHg}$. Quando presente HIC refratária a fármacos e a procedimentos cirúrgicos, deve-se manter a $\mathrm{PaCO}_{2}<30 \mathrm{mmHg}$, com monitorização da $\mathrm{SjO}_{2}$ e do FSC. ${ }^{(17)}$

\section{Utilizaçáo de pressáo positiva expiratória final}

Entre os artigos sobre a PEEP, cinco incluem níveis va- riando de $0 \mathrm{a} 15 \mathrm{cmH}_{2} \mathrm{O}$ e sugerem que a PEEP pode ser utilizada, haja visto não ter havido incrementos estatisticamente significantes da PIC. ${ }^{(4,14,27,29,30)}$ Videtta et al. observaram aumento significativo da PIC em PEEP de 10 a $15 \mathrm{cmH}_{2} \mathrm{O}$, não havendo mudanças significativas na PPC. ${ }^{(28)}$ Gamberoni et al. evidenciaram mudanças na mecânica respiratória devido ao $\mathrm{TCE}^{(26)}$ e três artigos mostraram que, em pacientes com baixa complacência pulmonar, a utilização da PEEP não produz variaçôes significativas na PIC (Quadro 2). ${ }^{(14,18,25)}$

\section{Quadro 2 - Resultados da utilizaçáo da pressáo positiva expiratória final}

\begin{tabular}{|c|c|c|c|c|}
\hline Referência & Estudo & Método & Resultados & Conclusáo \\
\hline $\begin{array}{l}\text { Dutton RP, } \\
\text { Mccunn M. } \\
2003^{(4)}\end{array}$ & $\mathrm{R}$ & $\begin{array}{l}\text { Uso da PEEP com monitorização } \\
\text { da PIC e PPC. }\end{array}$ & $\begin{array}{l}\text { O ensinamento clássico de nấo usar } \\
\text { ou usar baixos níveis de PEEP para } \\
\text { prevenir a elevaçấo da PIC é inapro- } \\
\text { priado, pois pode haver falha na cor- } \\
\text { reçáo da hipoxemia. }\end{array}$ & $\begin{array}{l}\text { PEEP não eleva a PIC, nem } \\
\text { deprime a PPC, podendo } \\
\text { ocorrer reduçáo da PIC pela } \\
\text { melhor oxigenaçáo cerebral, } \\
\text { quando em adequado volume } \\
\text { pulmonar. }\end{array}$ \\
\hline $\begin{array}{l}\text { Deem S. } \\
2006^{(14)}\end{array}$ & $\mathrm{R}$ & $\begin{array}{l}\text { Redução do } \mathrm{Vt} \text { para } 4-6 \mathrm{~mL} / \mathrm{Kg} \\
\text { e pressão de platô }<30 \mathrm{cmH}_{2} \mathrm{O} \text {, } \\
\text { sendo observada mudanças na } \\
\text { PIC, } \mathrm{PPC} \text { e } \mathrm{PaCO}_{2} \text {. }\end{array}$ & $\begin{array}{l}\text { A PEEP aumenta a pressão intratorá- } \\
\text { cica e reduz o fluxo venoso ao coraçáo, } \\
\text { resultando no aumento da pressão } \\
\text { venosa jugular, e assim a PIC, PPC e } \\
\mathrm{PaCO}_{2} \text { tornam-se elevadas. }\end{array}$ & $\begin{array}{l}\text { A PEEP deve ser aplicada cui- } \\
\text { dadosamente em pacientes } \\
\text { com TCE agudo, porém não } \\
\text { deve ser evitada na necessidade } \\
\text { de se manter uma oxigenaçáo } \\
\text { adequada. }\end{array}$ \\
\hline $\begin{array}{l}\text { Caricato A, et } \\
\text { al. } 2005^{(18)}\end{array}$ & $\mathrm{E}$ & $\begin{array}{l}\text { Investigação de } 21 \text { pacientes coma- } \\
\text { tosos com TCE grave ou hemor- } \\
\text { ragia subaracnóide com monitori- } \\
\text { zação da PIC, em VM com PEEP, } \\
\text { divididos em } 2 \text { grupos: (A) } 13 \\
\text { pacientes com complacência pul- } \\
\text { monar normal e (B) } 8 \text { com baixa } \\
\text { complacência. Aplicadas PEEP de } \\
0,5,8 \text { e } 12 \mathrm{cmH}_{2} \mathrm{O} \text { e mensuradas: } \\
\text { pressão jugular, PVC, PIC, PPC, } \\
\text { complacência cerebral, VmMCA } \\
\text { e } \mathrm{SjO}_{2} \text {. }\end{array}$ & $\begin{array}{l}\text { No grupo A, a elevação da PEEP de } 0 \\
\text { a } 12 \mathrm{cmH}_{2} \mathrm{O} \text {, aumentou significativa- } \\
\text { mente a PVC e a pressão jugular, mas } \\
\text { reduziu a PAM, PPC e VmMCA. } \\
\text { No } \mathrm{B} \text {, não houve variaçóes significati- } \\
\text { vas. A PIC e a complacência cerebral } \\
\text { não se modificaram nos dois grupos. } \\
\text { Porém, houve um decréscimo na } \\
\mathrm{SjO}_{2} \text {. }\end{array}$ & $\begin{array}{l}\text { Em pacientes com baixa com- } \\
\text { placência pulmonar, náo houve } \\
\text { efeito na hemodinâmica sistê- } \\
\text { mica e cerebral. Monitorização } \\
\text { da complacência pulmonar } \\
\text { pode servir para evitar os efei- } \\
\text { tos deletérios da PEEP no sist. } \\
\text { intracraniano em pacientes } \\
\text { com complacência normal. }\end{array}$ \\
\hline $\begin{array}{l}\text { Helmy A, } \\
\text { Vyzcaychipi } \\
\text { M, Gupta AK. } \\
2007^{(25)}\end{array}$ & $\mathrm{R}$ & $\begin{array}{l}\text { Discussáo do manuseio intensivo } \\
\text { do TCE grave. }\end{array}$ & $\begin{array}{l}\text { Não há contra-indicação absoluta ao } \\
\text { uso da PEEP em pacientes hipoxêmi- } \\
\text { cos, a menos que a pressão intratorá- } \\
\text { cica eleve a PIC. }\end{array}$ & $\begin{array}{l}\text { A PEEP está indicada em pa- } \\
\text { cientes com injúria pulmonar. }\end{array}$ \\
\hline $\begin{array}{l}\text { Gamberoni C, } \\
\text { et al. } 2002^{(26)}\end{array}$ & $\mathrm{E}$ & $\begin{array}{l}\text { Análise das mudanças na mecâ- } \\
\text { nica respiratória e os efeitos da } \\
\text { PEEP em } 10 \text { indivíduos sadios, } \\
10 \text { pacientes com injúria cerebral } \\
\text { sem lesão pulmonar e } 10 \text { pacien- } \\
\text { tes com injúria cerebral com lesão } \\
\text { pulmonar. }\end{array}$ & $\begin{array}{l}\text { A elastância do sistema respiratório, } \\
\text { da parede torácica e a resistência má- } \\
\text { xima pulmonar se elevaram em in- } \\
\text { júria cerebral independente de lesão } \\
\text { pulmonar. Em injúria cerebral e lesão } \\
\text { pulmonar, a PEEP de } 15 \mathrm{cmH}_{2} \mathrm{O} \text { ele- } \\
\text { vou a elastância do sistema respirató- } \\
\text { rio, da parede torácica e da resistência } \\
\text { máxima pulmonar. }\end{array}$ & $\begin{array}{l}\text { Em injúria cerebral, a mecâni- } \\
\text { ca respiratória torna-se altera- } \\
\text { da. A PEEP não tem efeito na } \\
\text { melhora da função respiratória } \\
\text { na lesão pulmonar causada por } \\
\text { pneumonia associada à venti- } \\
\text { lação. }\end{array}$ \\
\hline
\end{tabular}


Quadro 2 - Continuação

\begin{tabular}{|c|c|c|c|c|}
\hline Referência & Estudo & Método & Resultados & Conclusáo \\
\hline $\begin{array}{l}\text { Georgiadis D, } \\
\text { et al. } 2001^{(27)}\end{array}$ & $\mathrm{E}$ & $\begin{array}{l}\text { Aumento da PEEP }(4,8,12 \text { e } 4 \\
\left.\mathrm{cmH}_{2} \mathrm{O}\right) \text { em } 20 \text { pacientes por } 5 \\
\text { min, em cada valor, e mensurada } \\
\text { PAM, PIC, frequência respiratória } \\
\text { e VmMCA. }\end{array}$ & $\begin{array}{l}\text { A PPC variou significativamente. Em } \\
15 \text { pacientes, os parâmetros perma- } \\
\text { neceram estáveis. Em 7, um pequeno } \\
\text { aumento na PIC foi relatado na PAM. } \\
\text { Em 3, o aumento da PEEP gerou de- } \\
\text { créscimo da PAM e VmMCA, com } \\
\text { PIC estável ou leve declínio. }\end{array}$ & $\begin{array}{l}\text { Incremento da PEEP até } 12 \\
\text { mmHg não tem influência sig- } \\
\text { nificativa nos valores da PIC. } \\
\text { A aplicação da PEEP é segura, } \\
\text { permitindo que a PAM seja } \\
\text { mantida. }\end{array}$ \\
\hline $\begin{array}{l}\text { Videtta W, et } \\
\text { al. } 2002^{(28)}\end{array}$ & $\mathrm{E}$ & $\begin{array}{l}\text { Investigar a PIC e a PPC em dife- } \\
\text { rentes níveis de PEEP. } 20 \text { pacien- } \\
\text { tes em VM com monitorizaçáo da } \\
\text { PIC. } 10 \text { com TCE grave, } 5 \text { com } \\
\text { hemorragia intracerebral espontâ- } \\
\text { nea e } 5 \text { com hemorragia subarac- } \\
\text { nóidea. A PEEP foi elevada de } 5 \\
\text { até } 15 \mathrm{cmH} \mathrm{H}_{2} \mathrm{O} \text {, de } 5 \mathrm{em} 5 \mathrm{cmH}_{2} \mathrm{O} \text {. } \\
\text { Após, no mínimo, } 10 \text { minutos de } \\
\text { permanência, foram mensuradas a } \\
\text { PIC e PPC. }\end{array}$ & $\begin{array}{l}\text { A PEEP de } 10 \text { a } 15 \mathrm{cmH}_{2} \mathrm{O} \text { produziu } \\
\text { um significante aumento da PIC (mé- } \\
\text { dia de }+11,6 \mathrm{mmHg} \text { e }+14,6 \mathrm{mmHg} \\
\text { respectivamente), porém mudanças } \\
\text { insignificantes na PPC. }\end{array}$ & $\begin{array}{l}\text { A elevação da PIC é um efei- } \\
\text { to normal ao incremento da } \\
\text { PEEP, devendo-se atentar para } \\
\text { mudanças na PPC. }\end{array}$ \\
\hline $\begin{array}{l}\text { Huynh T, et } \\
\text { al. } 2002^{(29)}\end{array}$ & $\mathrm{E}$ & $\begin{array}{l}\text { Incremento gradual da PEEP em } \\
3 \text { grupos aleatórios de pacientes } \\
\text { com TCE grave e disfunção pul- } \\
\text { monar: } \\
\text { (1) } \quad 0 \text { a } 5 \mathrm{cmH}_{2} \mathrm{O} \\
\text { (2) } \quad 6 \text { a } 10 \mathrm{cmH}_{2} \mathrm{O} \\
\text { (3) } 11 \text { a } 15 \mathrm{cmH}_{2} \mathrm{O}\end{array}$ & $\begin{array}{l}\text { Houve decréscimo na PIC de: } \\
\text { (1) } 5,9 \pm 0,1 \mathrm{mmHg} \\
(2) \quad 8,3 \pm 0,2 \mathrm{mmHg} \\
(3) \\
12,0 \pm 0,3 \mathrm{mmHg}\end{array}$ & $\begin{array}{l}\text { A estratégia de incremento da } \\
\text { PEEP não está associada com } \\
\text { o comprometimento do trans- } \\
\text { porte de } \mathrm{O}_{2} \text { nem na exacerba- } \\
\text { ção da } \mathrm{HIC} \text {. }\end{array}$ \\
\hline $\begin{array}{l}\text { Mascia L, } \\
\text { Majorano M. } \\
2000^{(30)}\end{array}$ & $\mathrm{R}$ & $\begin{array}{l}\text { Manuseio ventilatório no TCE } \\
\text { agudo e os efeitos da PEEP. }\end{array}$ & $\begin{array}{l}\text { Os efeitos no FSC dependerão da } \\
\text { complacência intracraniana e do valor } \\
\text { absoluto da PIC, além dos efeitos he- } \\
\text { modinâmicos com a melhora da injú- } \\
\text { ria pulmonar. }\end{array}$ & $\begin{array}{l}\text { A PIC não irá se alterar, quan- } \\
\text { do o incremento da PVC pela } \\
\text { PEEP estiver abaixo do valor } \\
\text { da PIC. }\end{array}$ \\
\hline
\end{tabular}

$\mathrm{R}$ - revisão; E - ensaio clínico; $\mathrm{PPC}$ - pressão de perfusão cerebral; PEEP - pressão expiratória final positiva; $\mathrm{Vt}$ - volume corrente; VM - ventitação mecânica; $\mathrm{PaCO}_{2}$ - pressão parcial de gás carbônico; PVC - pressão venosa central; PAM - pressão arterial média; PIC - pressão intracranina; HIC - hipertensão intracraniana; FSC - fluxo sanguíneo cerebral; TCE - traumatismo cranioencefálico; SjO2 - saturaçáo venosa jugular de oxigênio.

Graus moderados da PEEP, iguais a $15 \mathrm{cmH}_{2} \mathrm{O}$, ou mesmo níveis mais altos podem com segurança serem usados na conduta de pacientes com lesáo cerebral, particularmente quando em baixa complacência pulmonar. ${ }^{(26)}$ Foi observado e sugerido que o uso da PEEP até $12 \mathrm{cmH}_{2} \mathrm{O}$ resultavam em um aumento insignificante da PIC. ${ }^{(27)}$

A aplicação da PEEP em níveis de 10 e $15 \mathrm{cmH}_{2} \mathrm{O}$ aumentou significativamente a PIC sem alteração importante na PPC, em pacientes com lesão pulmonar aguda (LPA). ${ }^{(28)}$

$\mathrm{O}$ aumento dos níveis da PEEP de 0 a $12 \mathrm{cmH}_{2} \mathrm{O}$ gera uma redução da PAM nos pacientes com complacência normal e esses mesmos valores em pacientes com baixa complacência não acarretam variaçóes significativas. Portanto, a complacência respiratória normal é um dos fatores que auxiliam na transmissão dos efeitos deletérios da PEEP ao sistema intracraniano. ${ }^{(18)}$
O controle cuidadoso da pressão de platô, até $30 \mathrm{cmH}_{2} \mathrm{O}$, deve ser uma prática razoável com devida fiscalização da PIC e da PPC quando houver elevação da $\mathrm{PaCO}_{2} \cdot{ }^{(14)}$ Não usar ou usar baixos níveis de PEEP para evitar uma elevação da PIC é inadequado, pois deixa de corrigir a hipoxemia, que pode diminuir a PIC por uma melhor oxigenação cerebral. ${ }^{(4,25)} \mathrm{O}$ efeito da PEEP na circulação cerebral depende da complacência intracraniana e do valor absoluto da PIC. A PIC não será afetada enquanto estiver acima da PVC gerada pela PEEP. ${ }^{(30)}$

Devido a problemas metodológicos, os estudos epidemiológicos sobre TCE são escassos, sendo essa dificuldade encontrada inclusive nos países mais desenvolvidos. ${ }^{(31)} \mathrm{O}$ manuseio ventilatório embasado em conhecimentos científicos nesses pacientes torna-se necessário, podendo interferir no prognóstico dos mesmos. 


\section{CONCLUSÁO}

Embasado em estudos científicos, assim como no melhor entendimento da fisiopatologia do TCE e as suas repercussóes, a hiperventilação profilática nas primeiras 24 horas, no intuito de se obter uma reduçáo da PIC por vasoconstricçáo cerebral, pode levar a um aumento da área cerebral lesionada por hipoperfusão tecidual. A hiperventilação prolongada deve ser evitada na ausência de PIC elevada. Já a hiperventilação otimizada, em curto período, parece ser a técnica mais promissora no controle da PIC e PPC.

A elevação da PEEP, limitada em $15 \mathrm{cmH}_{2} \mathrm{O}$, pode ser aplicada de forma consciente com objetivo na melhoria da oxigenaçáo alveolar e elevação da $\mathrm{SaO}_{2}$ na injúria pulmonar, permitindo assim uma maior oxigenação cerebral e melhora da complacência pulmonar.

\section{ABSTRACT}

The study intended to make a critical review on use of pulmonary hyperventilation maneuvers and the different positive end-expiratory pressures applied to traumatic brain injury patients. As a reference were used publications in English, Spanish and Portuguese, contained in the following databases: MedLine, SciELO and LILACS, from 2000 to 2007, we included all studies about the use of pulmonary hyperventilation maneuvers and the different positive end-expiratory levels used for adult patients with brain injury at acute or chronic stage. Thirty one trials were selected, 13 about pulmonary hyperventilation, as prophylaxis, prolonged or optimized and 9 shows the levels of positive end-expiratory pressures used, ranging from 0 to $15 \mathrm{cmH}_{2} \mathrm{O}$. The prophylactic hyperventilation maneuver in the first 24 hours can lead to an increase of cerebral ischemia; the prolonged hyperventilation must be avoided if intracranial pressure did not increase; however optimized hyperventilation seems to be the most promising technique for control of the intracranial pressure and cerebral perfusion pressure; the rise of the positive end-expiratory pressure, up to $15 \mathrm{cmH}_{2} \mathrm{O}$, can be applied in a conscientious form aiming to increase arterial oxygen saturation in lung injury.

Keywords: Brain trauma; Intracranial hypertension; Intracranial pressure; Positive-pressure respiration; Hyperventilation

\section{REFERÊNCIAS}

1. Werner C, Engelhard K. Pathophysiology of traumatic brain injury. Br J Anaesth. 2007;99(1):4-9. Review.

2. Ferrari D. Trauma: questão de saúde pública. RE Rev Emergê Clín . 2006;5:12-4.

3. Gottesman RF, Komotar R, Hillis AE. Neurologic aspects of traumatic brain injury. Int Rev Psychiatry. 2003;15(4):302-9.

4. Dutton RP, McCunn M. Traumatic brain injury. Curr Opin Crit Care. 2003; 9(6):503-9. Review.

5. Ribas GC. Traumatismo cranioencefálico. In: Nitrini R, Bacheschi LA. A neurologia que todo médico deve saber. 2a. ed. São Paulo: Atheneu; 2003. p. 189-203.

6. Paranhos JLR, Moura AD. Traumatismo cranioencefálico. In: David CM, editor. Medicina intensiva. Rio de Janeiro: Revinter; 2004. p. 770.

7. Braunwald E, Fauci AS, Kasper DL, Hauser SL, Longo DL, Jameson JL, editors. Harrison medicina interna. 16a. ed. Rio de Janeiro: McGraw-Hill; 2006. v. 2.

8. Zink BJ. Traumatic brain injury outcome: concepts for emergency care. Ann Emerg Med. 2001;37(3):318-32.

9. André C, Freitas GR, organizadores. Terapia intensiva em neurologia e neurocirurgia. Rio de Janeiro: Revinter; 2002.

10. Belda FJ, Aguilar G, Soro M, Maruenda A. Manejo ventilatório del paciente con traumatismo craneoencefálico grave. Rev Esp Anestesiol Reanim. 2004;51(3):143-50.
11. Tavares MP. Tratamento clínico do traumatismo crânioencefálico [ Internet]. Disponível em: <http://www.medstudents.com.br/neuroc/neuroc1.htm > Acesso em: 27 jul. 2006.

12. Giugno KM, Maia TR, Kunrath CL, Bizzi JJ. Tratamento da hipertensão intracraniana. J Pediatr (Rio J). 2003;79(4):287-96.

13. Stocchetti N, Maas AI, Chieregato A, van der Plas AA. Hyperventilation in head injury: a review. Chest. 2005;127(5):1812-27.

14. Deem S. Management of acute brain injury and associated respiratory issues. Respir Care. 2006;51(4):357-67.

15. Vincent JL, Berré J. Primer on medical management of severe brain injury. Crit Care Med. 2005;33(6):1392-9. Erratum in: Crit Care Med. 2005;33(9):2157. Comment in: Crit Care Med. 2005;33(11):2723; author reply 2723.

16. Brain Trauma Foundation; American Association of Neurological Surgeons; Congress of Neurological Surgeons; Joint Section on Neurotrauma and Critical Care, AANS/ CNS, Bratton SL, Chestnut RM, Ghajar J, McConnell Hammond FF, Harris OA, Hartl R, Manley GT, Nemecek A, Newell DW, Rosenthal G, Schouten J, Shutter L, Timmons SD, Ullman JS, Videtta W, Wilberger JE, Wright DW. Guidelines for the management of severe traumatic brain injury. XIV. Hyperventilation. J Neurotrauma. 2007;24 Suppl 1:S87-90. Erratum in: J Neurotrauma. 2008;25(3):276-8. multiple author names added. 
17. GhajarJ.Traumaticbrain injury. Lancet. 2000;356(9233):9239. Review.

18. Caricato A, Conti G, Della Corte F, Mancino A, Santilli F, Sandroni C, et al. Effects of PEEP on the intracranial system of patients with head injury and subarachnoid hemorrhage: the role of respiratory system compliance. J Trauma. 2005;58(3):571-6.

19. Andrade FC, Andrade Júnior FC. Usos e abusos da hiperventilação nos traumatismos crânio-encefálicos graves. Arq Neuropsiquiatr. 2000;58(3A)648-55.

20. Falcão ALE, Araujo S, Dragosavac D, Terzi RGG, Thiesen R AS, Cintra EA, et al. Hemometabolismo cerebral: variaçóes na fase aguda do coma traumático. Arq Neuropsiquiat. 2000; 58(3B):877-82.

21. Marik PE, Varon J, Trask T. Management of head trauma. Chest. 2002;122(2):699-711.

22. Valadka AB, Robertson CS. Surgery of cerebral trauma and associated critical care. Neurosurgery. 2007;61(1 Suppl): 203-20; discussion 220-1.

23. Vender JR. Hyperventilation in severe brain injury revisited. Crit Care Med. 2000;28(9):3361-2.

24. Brain Trauma Foundation. Management and prognosis of severe traumatic traumatic brain injury. New York (NY): The Brain Trauma Foundation, 2000.

25. Helmy A, Vizcaychipi M, Gupta AK. Traumatic brain injury: intensive care management. Br J Anaesth. 2007;99(1):3242. Review.

26. Gamberoni C, Colombo G, Aspesi M, Mascheroni C, Severgnini $P$, Minora $G$, et al. Respiratory mechanics in brain injured patients. Minerva Anestesiol. 2002;68(4):291-6.

27. Georgiadis D, Schwarz S, Baumgartner RW, Veltkamp R, Schwab S. Influence of positive end-expiratory pressure on intracranial pressure and cerebral perfusion pressure in patients with acute stroke. Stroke. 2001;32(9):2088-92.

28. Videtta W, Villarejo F, Cohen M, Domeniconi G, Santa Cruz R, Pinillos O, et al. Effects of positive end-expiratory pressure on intracranial pressure and cerebral perfusion pressure. Acta Neurochir Suppl. 2002;81:93-7.

29. Huynh T, Messer M, Sing RF, Miles W, Jacobs DG, Thomason MH. Positive end-expiratory pressure alters intracranial and cerebral perfusion pressure in severe traumatic brain injury. J Trauma. 2002;53(3):488-92; discussion 492-3.

30. Mascia L, Majorano M. Mechanical ventilation for patients with acute brain injury. Curr Opin Crit Care. 2000;6(1):52-6.

31. Koizumi MS, Lebrão ML, Mello-Jorge MHP, Primerano V. Morbimortalidade por traumatismo crânio-encefálico no município de Sáo Paulo, 1997. Arq Neuropsiquiatr. 2000;58(1):81-9. 